\title{
Effective mass: A new concept in stellar astrophysics based on the internal rotation, and its place in the A- and B-star puzzle
}

\author{
Mutlu Yıldız \\ Ege University, Dept. of Astronomy and Space Sciences, Bornova, 35100 Izmir, Turkey \\ email: yildiz@astronomy.sci.ege.edu.tr
}

\begin{abstract}
Putting their chemically peculiarities aside, Ap and Am stars have many common properties. The discriminating property between them could be the rotation of their interiors beneath their slowly rotating surface. In the PV Cas (HD 240208) binary system whose light curve shows Ap-like variation, the agreement between the theoretical and observed apsidal advance rate is satisfied only with differentially rotating models of the component stars in which rapid rotation is extended almost to the surface. Thus, it seems that there is a steep rotation rate gradient near the surface of the Ap stars. The conclusion we reach in the analysis of PV Cas system leads us to introduce the effective mass as a new conceptual tool in stellar astrophysics: $M_{\text {eff }}=M_{\star}(1-\bar{\Lambda})^{1.75}$, where $M_{\star}$ and $\bar{\Lambda}$ are mass of the star and average of ratio of centrifugal to the gravitational acceleration throughout the model, respectively. We find that the effective mass of PV Cas A whose true mass is $2.82 \pm 0.05 M_{\odot}$ is $2.6 M_{\odot}$ for both solar and metal rich compositions.
\end{abstract}

Keywords. Stars: rotation, stars: interiors, stars: evolution, stars: early-type, binaries: eclipsing, stars: individual (PV Cas)

\section{Introduction}

The conventional conceptual tools for our understanding of the stellar structure and the evolution fail to explain many important observed properties of A-stars. In this respect, the assumption of no or solid-body rotation may be the most important deficiency in the standard models. In fact, differential rotation with depth should be expected as a natural result of contraction, at least for some time, provided that the angular momentum transfer in the stellar interior is not a sudden process. The evolution of such a differential rotation depends on the details of angular momentum transfer layer by layer. Although we have no exact information about this transfer process so far, there are strong evidences that the internal regions of A- and B-type stars are rotating much more rapidly than their outer regions (see Ylldız 2005).

In this study, we further discuss the effect of rotation on the model properties and explain some basic irregularities arising from the rapid rotation of the inner regions, by introducing the effective mass concept. The main motivation for doing this comes from the comparison of the differentially rotating model of PV Cas A with the non-rotating model of 2.55 $M_{\odot}$. In Figure 1, these models (with metal rich composition) are plotted in the HR diagram: While the former is represented by $\star, \times$ marks the evolutionary track of the latter. For further comparison, the non-rotating model of $2.82 M_{\odot}$ is also plotted in this figure $(\diamond)$. Differential rotation of this kind makes the structure and evolution of PV Cas A with mass $2.82 M_{\odot}$ very close to those of the non-rotating model of $2.55 M_{\odot}$. Thus, a differentially rotating model of a certain mass is structured and evolves as though it has somewhat a reduced mass. 


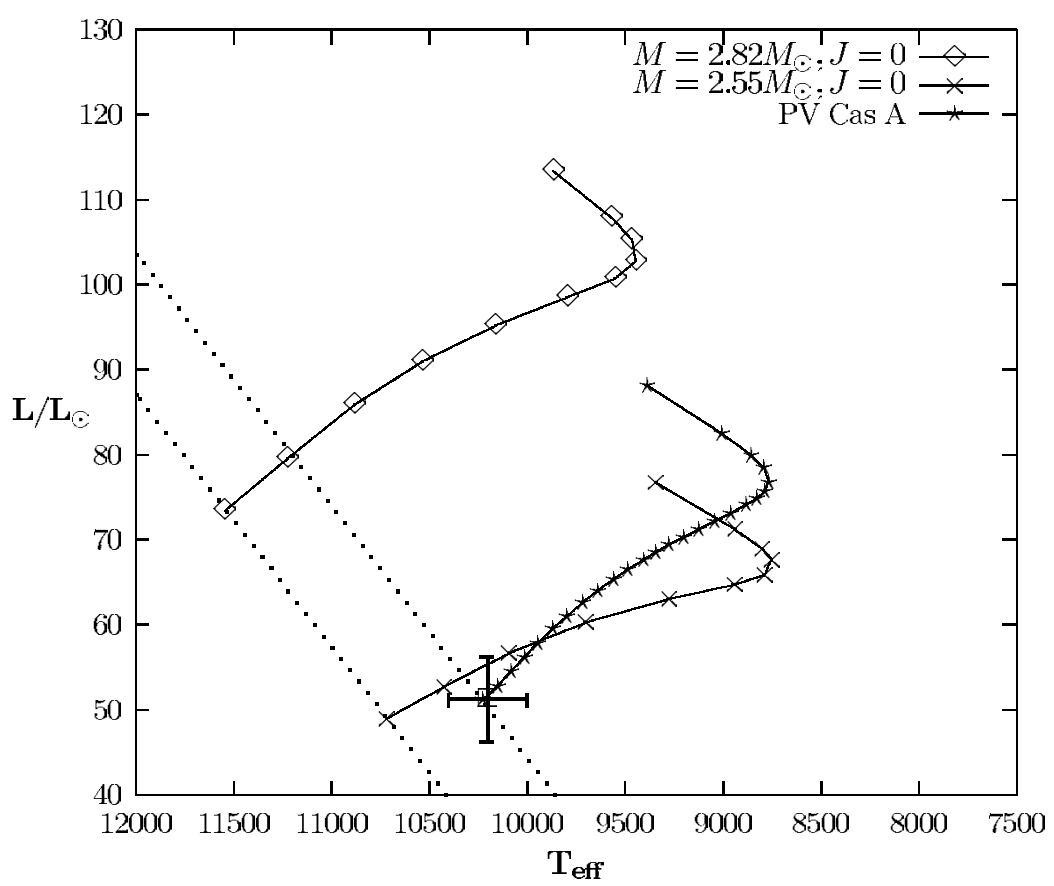

Figure 1. The observed luminosity of PV Cas A is sufficiently low that it has the same luminosity level as the non-rotating model of $2.55 M_{\odot}(\times)$ rather than of $2.82 M_{\odot}(\diamond)$. Differential rotation is required to fit model of PV Cas A $(\star)$ to the observations. Two parallel dotted lines show the ZAMS lines for the non-rotating (lower) and differentially rotating models (upper).

Note also that the difference between the effective temperatures of the two ZAMS lines (one for the non-rotating models and the other for the differentially rotating models similar to that for PV Cas A), for a given luminosity, is the same as the difference found by Hubrig et al. (2000) between the effective temperatures of the magnetic Ap and normal stars.

In this study, the results are essentially based on a rich variety of rotating models constructed for the mass of the primary component PV Cas (Ylldız 2004). The rest of the paper is organized as follows: In Sect. 2, the basic effects of rotation are discussed and some relations between the quantities of non-rotating models and models with solid-body rotation are presented. An analytic expression for the homogeneous mass distribution is derived for effective mass in Sect. 3, while we obtain a similar expression for a more realistic case in Sect. 4. Finally, Sect. 5 contains some conclusions.

\section{Basic effects of rotation}

The mean effect of rotation on hydrostatic equilibrium in terms of rotation parameter $\Lambda_{\mathrm{r}}$ can be given as (Kippenhahn et al. 1970)

$$
\frac{d P}{d r}=-\frac{G M_{r} \rho}{r^{2}}\left(1-\Lambda_{\mathrm{r}}\right)
$$

where

$$
\Lambda_{\mathrm{r}}=\frac{2}{3} \frac{\Omega^{2} r^{3}}{G M_{r}}
$$


Due to the radial component of centrifugal acceleration, which is opposite to gravity, the central regions feel less mass than the true mass of the overlying regions. As a result the central temperature of a rotating model is always less than that of its non-rotating counterpart. Therefore, the former is always fainter than the latter. In the case of solidbody rotation, for example, we derive that luminosity of the rotating model can be given in terms of luminosity of the non-rotating model $\left(L_{\mathrm{o}}\right)$ and the value of the rotational parameter at the surface $\left(\Lambda_{\mathrm{s}}\right)$

$$
L=\frac{L_{\mathrm{o}}}{\left(1+\Lambda_{\mathrm{s}}\right)^{0.25}} .
$$

The effect of solid-body rotation on the radius, however, can be formulated from the model properties as

$$
R=R_{\mathrm{o}}\left(1+\Lambda_{\mathrm{s}}\right)^{0.45} .
$$

Our aim is to find similar relations for the luminosities and the radii of differentially rotating models. But, the radius is a more complicated function of the rotational profile than the luminosity. Now, we shall see how the central physical conditions of a model depend on its total mass and rotational properties.

\section{Effective mass for a homogeneous mass distribution}

All the overlying layers compress the central regions. However, rotation acts to reduce this effect. Then, central physical conditions result as though there were less mass in the upper layers than the true mass. To find a simple relation between the physical conditions at the center and the total mass, and also how rotation affects this relation, we assume a constant mass distribution. Using the equation of continuity and integrating Eq. (2.1) from the center to the surface, we get

$$
\frac{P_{\mathrm{c}}}{c \rho_{\mathrm{o}}^{4 / 3}}=\frac{\Re}{\mu} \frac{T_{\mathrm{c}}}{c \rho_{\mathrm{c}}^{1 / 3}}=\frac{3}{2} M_{\star}^{2 / 3}(1-\bar{\Lambda})
$$

where mean rotational parameter $\bar{\Lambda}$ is defined as

$$
\bar{\Lambda} \equiv \frac{2}{3 M_{\star}^{2 / 3}} \int_{0}^{M_{\star}} \frac{d M_{r}}{M_{r}^{1 / 3}} \Lambda_{\mathrm{r}} .
$$

For simplicity, in (3.1), we take only the gas pressure into consideration. Indeed, (3.1) describes implicitly the mass-luminosity relation in the case of rotation: The physical conditions at the center, where nuclear reactions are occurring, are determined by the total mass and $\bar{\Lambda}$, for a given chemical composition. Furthermore, these conditions could be very similar for two stars with very different masses, provided that their masses and mean rotational parameters give the same value for the right side of (3.1). We define effective mass in this context as

$$
M_{\mathrm{NR}} \equiv M_{\mathrm{eff}}(\bar{\Lambda}) \equiv M_{\star}(1-\bar{\Lambda})^{3 / 2}
$$

where $M_{\mathrm{NR}}$ is the corresponding mass for no rotation. That is to say, a model of mass $M$ with $\bar{\Lambda}=0.63$ has the same physical conditions at its center as the non-rotating model of mass $M / 2$.

\section{The effective mass of PV Cas A}

For a more realistic case than the homogeneous mass distribution, we construct models of mass $2.82 M_{\odot}$ with a variety of rotational properties. In Figure 2, luminosities of these 


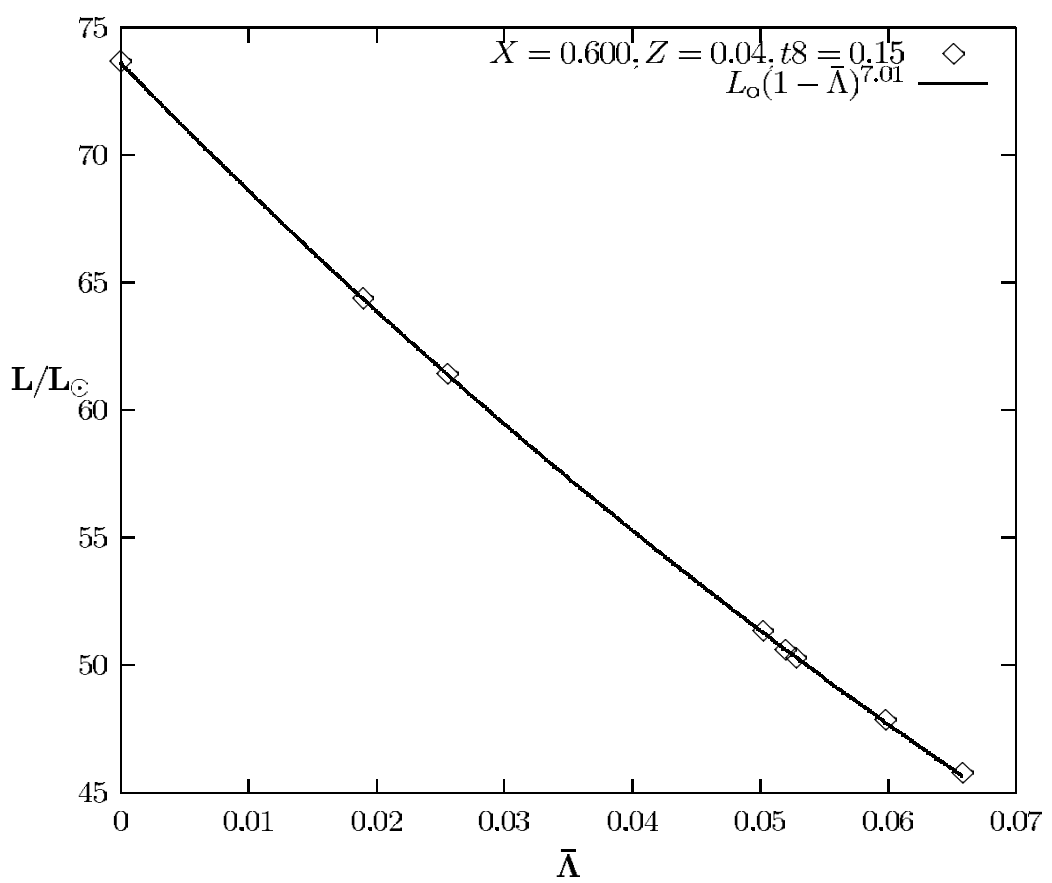

Figure 2. Luminosities of models of $2.82 M_{\odot}$ with a variety of rotational properties as a function of the average of rotational parameter $\bar{\Lambda}$.

models at $t=15 \mathrm{My}(\diamond)$ are plotted with respect to their mean rotational parameter $\bar{\Lambda}$. The solid line represents the fitting formulae $L_{\mathrm{o}}(1-\bar{\Lambda})^{7.01}$, where $L_{\mathrm{o}}$ is luminosity of the non-rotating model. All points are on this curve.

To find an expression for the effective mass, we use the mass-luminosity relation derived from the non-rotating models of $2.82 M_{\odot}$ and $2.55 M_{\odot}$ with metal rich composition; $L=c M^{4}$. Then,

$$
M_{\mathrm{eff}}(\bar{\Lambda})=M_{\star}(1-\bar{\Lambda})^{7.01 / 4}
$$

For the differentially rotating model with a metal rich composition given in Figure 1, using (4.1), we find that $M_{\text {eff }}=2.58 M_{\odot}$. Similarly, we can also compute the effective mass for the solar composition: $M_{\text {eff }}=2.60 M_{\odot}$. Thus these values for the effective mass are almost the same.

As it is emphasized above, the effective mass determines the evolution of stars as well as their structures. Comparison of the MS lifetimes of the models shows this very clearly: The MS lifetime of non-rotating model of $2.82 M_{\odot}$ is $280 \mathrm{My}$, while the non-rotating model of $2.57 M_{\odot}$ and the differentially rotating model of $2.82 M_{\odot}$ have the same MS lifetime (370 My).

The use of effective mass may also explain why the observed mass-luminosity relation for the PV Cas binary system is so low (Barembaum \& Etzel 1995):

$$
\frac{\Delta \log L}{\Delta \log M}=2.3 \text {. }
$$

The minimum value computed from the models of PV Cas A \& B for this relation is 4 . If we use the effective masses of PV Cas A \& B instead of true masses we get 3.6, very close to the theoretical value (4). 


\section{Conclusions}

There is strong evidence that the inner regions of some (if not all) early type stars are rotating very rapidly. We discussed the effects of such a rotation on the structure and the evolution of stars, by introducing effective mass concept. According to our findings concerning the calibrated model of PV Cas A, its effective mass is $10 \%$ less than its true mass, as a result of differential rotation. Another surprising result, pertaining to stellar evolution, is that the MS lifetime of a differentially rotating model is also determined by the effective mass.

Since the Ap-like variations are observed in the light curve of the PV Cas binary system, some or all of the Ap stars may have such a differential rotation. In that respect, maybe, ratio of the effective mass to the true mass is minimum for Ap stars with such rotational properties.

The effective mass concept also explains why the observed luminosity relation is so small for some eclipsing binaries in comparison with the minimum theoretical value. Furthermore, the effective mass may have also some cosmological implications.

\section{Acknowledgements}

D. Özlem Hürkal is thanked for reading the manuscript.

\section{References}

Barembaum, M.J., \& Etzel, P.B. 1995, AJ 109, 2680

Hubrig, S., North, P., \& Mathys, G. 2000, ApJ 539, 352

Kippenhahn, R., Meyer-Hofmeister, E., \& Thomas, H.-C.i 1970, A $\& A$ 5, 155

Yıldız, M. 2004, A\&\&A, submitted

Yıldız, M. 2005, These Proceedings, 81 